\title{
A NOTE ON THE DEGENERATE POLY-CAUCHY POLYNOMIALS AND NUMBERS OF THE SECOND KIND
}

\author{
HYE KYUNG KIM ${ }^{1}$ AND LEE-CHAE JANG ${ }^{2, *}$
}

\begin{abstract}
Kim(2015) introduced the degenerate Cauchy numbers of the second kind and gave some identities of them. In this paper, by using the modified polyexponential functions which are introduced by Kim-Kim(2019), we define the degenerate poly-Cauchy polynomials of the second kind and investigate some properties of them. Furthermore, we consider the degenerate unipoly-Cauchy polynomials of the second kind and discuss some properties of them.
\end{abstract}

\section{Introduction}

As is well known, the Cauchy polynomials $C_{n}(x)$ (or the Bernoulli polynomials of the second kind) are derived from the integral as follows:

$$
\int_{0}^{1}(1+t)^{x+y} d y=\frac{t}{\log (1+t)}(1+t)^{x}=\sum_{n=0}^{\infty} C_{n}(x) \frac{t^{n}}{n !}, \quad(\text { see }[1,14,15,19]) .
$$

When $x=0, C_{n}=C_{n}(0)$ are called the Cauchy numbers. In [22], the Daehee polynomials $D_{n}(x)$ are defined by the generating function to be

$$
\frac{\log (1+t)}{t}(1+t)^{x}=\sum_{n=0}^{\infty} D_{n}(x) \frac{t^{n}}{n !}, \quad(\text { see }[4,22]) .
$$

When $x=0, D_{n}=D_{n}(0)$ are called the Cauchy numbers. Kim [14] defined the degenerate Cauchy polynomials $C_{n, \lambda}(x)$ as follows:

$$
\begin{aligned}
\int_{0}^{1}\left(1+\log (1+\lambda t)^{\frac{1}{\lambda}}\right)^{x+y} d y & =\frac{\frac{1}{\lambda} \log (1+\lambda t)}{\log \left(1+\frac{1}{\lambda} \log (1+\lambda t)\right)}\left(1+\log (1+\lambda t)^{\frac{1}{\lambda}}\right)^{x} \\
& =\sum_{n=0}^{\infty} C_{n, \lambda}(x) \frac{t^{n}}{n !}, \quad(\text { see }[2,7,18,21]) .
\end{aligned}
$$

When $x=0, C_{n, \lambda}=C_{n, \lambda}(0)$ are the degenerate Cauchy numbers. The degenerate Cauchy polynomials $C_{n, \lambda, 2}(x)$ of the second kind are introduced in [14] as follows:

$$
\frac{t}{\log \left(1+\frac{1}{\lambda} \log (1+\lambda t)\right)}\left(1+\frac{1}{\lambda} \log (1+\lambda t)\right)^{x}=\sum_{n=0}^{\infty} C_{n, \lambda, 2}(x) \frac{t^{n}}{n !} \text {, (see [9, 10, 24] ). }
$$

2010 Mathematics Subject Classification. 11B83, 11S80.

Key words and phrases. polylogarithm functions, unipoly functions, Cauchy polynomials, polyCauchy polynomials, unipoly-Cauchy polynomials. 
When $x=0, C_{n, \lambda, 2}=C_{n, \lambda, 2}(0)$ are called the degenerate Cauchy polynomials of the second kind. Pyo-Kim-Kim [24] introduced the degenerate Cauchy polynomials $C_{n, \lambda, 3}(x)$ of the third kind as follows:

$$
\frac{\lambda\left((1+\lambda \log (1+t))^{\frac{1}{\lambda}}-1\right)}{\log (1+\lambda \log (1+t))}(1+\lambda \log (1+t))^{\frac{x}{\lambda}}=\sum_{n=0}^{\infty} C_{n, \lambda, 3}(x) \frac{t^{n}}{n !}, \quad(\text { see }[9,10,24])
$$

and also introduced the degenerate Cauchy polynomials $C_{n, \lambda, 4}(x)$ of the fourth kind as follows:

$$
\frac{\lambda t}{\log (1+\lambda \log (1+t))}(1+\lambda \log (1+t))^{\frac{x}{\lambda}}=\sum_{n=0}^{\infty} C_{n, \lambda, 4}(x) \frac{t^{n}}{n !}, \quad(\text { see }[11,12,23]) .
$$

It is well known that the Stirling numbers of the first kind are defined by

$$
(x)_{n}=\sum_{l=0}^{n} S_{1}(n, l) x^{l}, \quad(\text { see }[3,15]),
$$

where $(x)_{0}=1,(x)_{n}=x(x-1) \ldots(x-n+1),(n \geq 1)$. From (1.7), it is easily to see that

$$
\frac{1}{k !}(\log (1+t))^{k}=\sum_{n=k}^{\infty} S_{1}(n, k) \frac{t^{n}}{n !}, \quad(\text { see }[3,15]) .
$$

In the inverse expression to (1.7), for $n \geq 0$, the Stirling numbers of the second kind are defined by

$$
x^{n}=\sum_{l=0}^{n} S_{2}(n, l)(x)_{l}, \quad(\text { see }[4,5,19])
$$

From (1.10), it is easily to see that

$$
\frac{1}{k !}\left(e^{t}-1\right)^{k}=\sum_{n=k}^{\infty} S_{2}(n, k) \frac{t^{n}}{n !}, \quad(\text { see }[3,25]) .
$$

In this paper, with a motive similar to the degenerate poly-Bernoulli polynomails, we define the degenerate poly-Cauchy polynomials of the second kind by using the polyexponential functions, and investigate their properties. Furthermore, we consider the degenerate unipoly Cauchy polynomials of the second kind and discuss some identities of them. 


\section{The degenerate poly-Cauchy polynomials of the second kind}

For $k \in \mathbb{Z}$, it is well known that the polylogarithm function $L i_{k}(x)$ is defined by a power series in $x$ to be

$$
L i_{k}(x)=\sum_{n=1}^{\infty} \frac{x^{n}}{n^{k}}=x+\frac{x^{2}}{2^{k}}+\frac{x^{3}}{3^{k}}+\cdots, \quad(\text { see }[5,6,16,18]) .
$$

Kim-Kim [?] defined the polyexponential function, as an inverse to the polylogarithm function

$$
E i_{k}(x)=\sum_{n=1}^{\infty} \frac{x^{n}}{(n-1) ! n^{k}}, \quad(\text { see }[8,13,17,20])
$$

When $k=1$, by (2.2), we get $E i_{1}(x)=e^{x}-1$. In the viewpoint of (1.4) and (2.2), we define the degenerate poly-Cauchy polynomials of the second kind as follows:

$$
\frac{E i_{k}(\log (1+t))}{\log \left(1+\frac{1}{\lambda} \log (1+\lambda t)\right)}\left(1+\frac{1}{\lambda} \log (1+\lambda t)\right)^{x}=\sum_{n=0}^{\infty} C_{n, \lambda, 2}^{(k)}(x) \frac{t^{n}}{n !}
$$

When $x=0, C_{n, \lambda, 2}^{(k)}=C_{n, \lambda, 2}^{(k)}(0)$ are called the degenerate poly-Cauchy numbers of the second kind. For $k=1$, by (2.2), we note that

$$
\begin{aligned}
E i_{1}(\log (1+t)) & =\sum_{n=1}^{\infty} \frac{(\log (1+t))^{n}}{(n-1) ! n} \\
& =e^{\log (1+t)}-1=t .
\end{aligned}
$$


By (2.3) and (2.4), we see that $C_{n, \lambda, 2}^{(1)}=C_{n, \lambda, 2}$. From (2.3) with $x=0$, we observe that

$$
\begin{aligned}
& \sum_{n=0}^{\infty} C_{n, \lambda, 2}^{(k)} \frac{t^{n}}{n !}=\frac{E i_{k}(\log (1+t))}{\log \left(1+\frac{1}{\lambda} \log (1+\lambda t)\right)} \\
& =\frac{t}{\log \left(1+\frac{1}{\lambda} \log (1+\lambda t)\right)} \frac{1}{t} \sum_{m=1}^{\infty} \frac{(\log (1+t))^{m}}{(m-1) ! m^{k}} \\
& =\frac{t}{\log \left(1+\frac{1}{\lambda} \log (1+\lambda t)\right)} \frac{1}{t} \sum_{m=0}^{\infty} \frac{(\log (1+t))^{m+1}}{(m+1) !(m+1)^{k-1}} \\
& =\frac{t}{\log \left(1+\frac{1}{\lambda} \log (1+\lambda t)\right)} \frac{1}{t} \sum_{m=0}^{\infty} \frac{1}{(m+1)^{k-1}} \sum_{l=m+1}^{\infty} S_{1}(l, m+1) \frac{t^{l}}{l !} \\
& =\frac{t}{\log \left(1+\frac{1}{\lambda} \log (1+\lambda t)\right)} \sum_{m=0}^{\infty} \frac{1}{(m+1)^{k-1}} \sum_{l=m}^{\infty} S_{1}(l+1, m+1) \frac{t^{l}}{(l+1) !} \\
& =\left(\sum_{s=0}^{\infty} C_{s, \lambda, 2} \frac{t^{s}}{s !}\right)\left(\sum_{l=0}^{\infty} \sum_{m=0}^{l} \frac{1}{(m+1)^{k-1}} \frac{S_{1}(l+1, m+1)}{l+1} \frac{t^{l}}{l !}\right) \\
& =\sum_{n=0}^{\infty}\left(\sum_{l=0}^{n} \sum_{m=0}^{l}\left(\begin{array}{l}
l \\
l
\end{array}\right) C_{n-l, \lambda, 2} \frac{S_{1}(l+1, m+1)}{(l+1)(m+1)^{k-1}}\right) \frac{t^{n}}{n !} .
\end{aligned}
$$

Therefore, by (2.5), we obtain the following theorem.

Theorem 2.1. For $n \geq 0, k \in \mathbb{Z}$, we have

$$
C_{n, \lambda, 2}^{(k)}=\sum_{l=0}^{n} \sum_{m=0}^{l}\left(\begin{array}{l}
n \\
l
\end{array}\right) C_{n-l, \lambda, 2} \frac{S_{1}(l+1, m+1)}{(l+1)\left((m+1)^{k-1}\right.} .
$$

Let us take $k=1$. From (2.6), we get

$$
\sum_{l=1}^{n} \sum_{m=0}^{l}\left(\begin{array}{l}
n \\
l
\end{array}\right) C_{n-l, \lambda, 2} \frac{S_{1}(l+1, m+1)}{(l+1)}=0 .
$$

In [5], it is well known that

$$
\frac{d}{d x} E i_{k}(\log (1+x))=\frac{1}{(1+x) \log (1+x)} E i_{k-1}(\log (1+x)) .
$$

From (2.8), we note that

$$
\begin{aligned}
& E i_{k}(\log (1+x))=\int_{0}^{x} \frac{1}{(1+t) \log (1+t)} E i_{k-1}(\log (1+t)) d t \\
& =\int_{0}^{x} \frac{1}{(1+t) \log (1+t)} \underbrace{\int_{0}^{t} \frac{1}{(1+t) \log (1+t)} \int_{0}^{t} \cdots \int_{0}^{t} \frac{t}{(1+t) \log (1+t)} d t d t \cdots d t .}_{(k-2) \text { times }}
\end{aligned}
$$


It is well known that

$$
\frac{t}{(1+t) \log (1+t)}=\sum_{l=0}^{\infty} B_{l}^{(l)} \frac{t^{l}}{l !}
$$

From (2.4), (2.9), and (2.10), we get

$$
\begin{gathered}
\sum_{n=0}^{\infty} C_{n, \lambda, 2}^{(k)} \frac{x^{n}}{n !}=\frac{E i_{k}(\log (1+x))}{\log \left(1+\frac{1}{\lambda} \log (1+\lambda x)\right)} \\
=\frac{1}{\log \left(1+\frac{1}{\lambda} \log (1+\lambda x)\right)} \int_{0}^{x} \frac{1}{(1+t) \log (1+t)} E i_{k-1}(\log (1+t)) d t \\
=\frac{1}{\log \left(1+\frac{1}{\lambda} \log (1+\lambda x)\right)} \int_{0}^{x} \frac{1}{(1+t) \log (1+t)} \\
\quad \times \underbrace{\int_{0}^{t} \frac{1}{(1+t) \log (1+t)} \int_{0}^{t} \cdots \int_{0}^{t} \frac{t}{(1+t) \log (1+t)} d t \cdots d t d t}_{(k-2) \text { times }}
\end{gathered}
$$

Let us take $k=2$. Then we have

$$
\begin{aligned}
& \sum_{n=0}^{\infty} C_{n, \lambda, 2}^{(2)} \frac{t^{n}}{n !} \\
& =\frac{1}{\log \left(1+\frac{1}{\lambda} \log (1+\lambda x)\right)} \int_{0}^{x} \frac{t}{(1+t) \log (1+t)} d t \\
& =\frac{1}{\log \left(1+\frac{1}{\lambda} \log (1+\lambda x)\right)} \sum_{l=0}^{\infty} \frac{B_{l}^{(l)}}{l !} \int_{0}^{x} t^{l} d t \\
& =\frac{x}{\log \left(1+\frac{1}{\lambda} \log (1+\lambda x)\right)} \sum_{l=0}^{\infty} \frac{B_{l}^{(l)}}{l+1} \frac{x^{l}}{l !} \\
& =\left(\sum_{m=0}^{\infty} C_{m, \lambda, 2} \frac{x^{m}}{m !}\left(\sum_{l=0}^{\infty} \frac{B_{l}^{(l)}}{l+1} \frac{x^{l}}{l !}\right)\right. \\
& =\sum_{n=0}^{\infty}\left(\sum_{l=0}^{n}\left(\begin{array}{c}
n \\
l
\end{array}\right) \frac{C_{n-l, \lambda, 2} B_{l}^{(l)}}{l+1}\right) \frac{x^{n}}{n !}
\end{aligned}
$$

Therefore, by (2.12), we obtain the following theorem.

Theorem 2.2. Let $n \geq 0$. Then we have

$$
C_{n, \lambda, 2}^{(2)}=\sum_{l=0}^{n}\left(\begin{array}{l}
n \\
l
\end{array}\right) \frac{C_{n-l, \lambda, 2} B_{l}^{(l)}}{l+1} .
$$


From (2.3), we observe that

$$
\begin{aligned}
& \sum_{n=0}^{\infty} C_{n, \lambda, 2}^{(k)}(x) \frac{t^{n}}{n !} \\
& =\frac{E i_{k}(\log (1+t))}{\log \left(1+\frac{1}{\lambda} \log (1+\lambda t)\right)}\left(1+\frac{1}{\lambda} \log (1+\lambda t)\right)^{x} \\
& =\left(\sum_{l=0}^{\infty} C_{l, \lambda, 2}^{(k)} \frac{t^{l}}{l !}\right)\left(\sum_{m=0}^{\infty}\left(\begin{array}{c}
x \\
m
\end{array}\right)\left(\frac{1}{\lambda} \log (1+\lambda t)\right)^{m}\right) \\
& =\left(\sum_{l=0}^{\infty} C_{l, \lambda, 2}^{(k)} \frac{t^{l}}{l !}\right)\left(\sum_{m=0}^{\infty}(x)_{m} \lambda^{-m} \sum_{s=m}^{\infty} S_{1}(s, m) \lambda^{s} \frac{t^{s}}{s !}\right) \\
& =\left(\sum_{l=0}^{\infty} C_{l, \lambda, 2}^{(k)} \frac{t^{l}}{l !}\right)\left(\sum_{s=0}^{\infty} \sum_{m=0}^{s}(x)_{m} \lambda^{-m} S_{1}(s, m) \lambda^{s} \frac{t^{s}}{s !}\right) \\
& =\sum_{n=0}^{\infty}\left(\sum_{l=0}^{n} \sum_{m=0}^{n-l}\left(\begin{array}{l}
n \\
l
\end{array}\right)_{l, \lambda, 2}^{(k)}(x)_{m} \lambda^{n-l-m} S_{1}(n-l, m)\right) \frac{t^{n}}{n !} .
\end{aligned}
$$

By comparing the coefficients on both sides of (2.14), we obtain the following theorem.

Theorem 2.3. Let $n \geq 0$ and $k \in \mathbb{Z}$. Then we have

$$
C_{n, \lambda, 2}^{(k)}(x)=\sum_{l=0}^{n} \sum_{m=0}^{n-l}\left(\begin{array}{l}
n \\
l
\end{array}\right) C_{l, \lambda, 2}^{(k)}(x)_{m} \lambda^{n-l-m} S_{1}(n-l, m) .
$$

\section{The degenerate unipoly-Cauchy polynomials of the second kind}

Let $p$ be any arithmetic function which is a real or complex valued function defined on the set of positive integers $\mathbb{N}$. Then Kim-Kim [5] defined the unipoly function attached to $p$ by

$$
u_{k}(x \mid p)=\sum_{n=1}^{\infty} \frac{p(n) x^{n}}{n^{k}}, \quad(k \in \mathbb{Z})
$$

It is well known that

$$
u_{k}(x \mid 1)=\sum_{n=1}^{\infty} \frac{x^{n}}{n^{k}}=L i_{k}(x)
$$

is ordinary polylogarithm function, and for $k \geq 2$,

$$
\frac{d}{d x} u_{k}(x \mid p)=\frac{1}{x} u_{k-1}(x \mid p)
$$

and

$$
u_{k}(x \mid p)=\int_{0}^{x} \frac{1}{t} \underbrace{\int_{0}^{t} \cdots \int_{0}^{t}}_{(k-2) \text { times }} \frac{1}{t} u_{1}(t \mid p) d t \cdots d t d t \quad \text { (see [?]) }
$$


By using (3.1), we define the degenerate unipoly-Cauchy polynomials of the second kind as follows:

$$
\frac{u_{k}(\log (1+t) \mid p)}{\log \left(1+\frac{1}{\lambda} \log (1+\lambda t)\right)}\left(1+\frac{1}{\lambda} \log (1+\lambda t)\right)^{\frac{x}{\lambda}}=\sum_{n=0}^{\infty} C_{n, \lambda, 2}^{(k, p)}(x) \frac{t^{n}}{n !} .
$$

When $x=0, C_{n, \lambda, 2}^{(k, p)}=C_{n, \lambda, 2}^{(k, p)}(0)$ are called the degenerate unipoly-Cauchy numbers of the second kind. Let us take $p(n)=\frac{1}{\Gamma(n)}$. Then we have

$$
\begin{aligned}
& \sum_{n=0}^{\infty} C_{n, \lambda, 2}^{(k, p)}(x) \frac{t^{n}}{n !}=\frac{u_{k}\left(\log (1+t) \mid \frac{1}{\Gamma} p\right)}{\log \left(1+\frac{1}{\lambda} \log (1+\lambda t)\right)}\left(1+\frac{1}{\lambda} \log (1+\lambda t)\right)^{\frac{x}{\lambda}} \\
& =\frac{1}{\log \left(1+\frac{1}{\lambda} \log (1+\lambda t)\right)} \sum_{m=1}^{\infty} \frac{(\log (1+t))^{m}}{m^{k}(m-1) !}\left(1+\frac{1}{\lambda} \log (1+\lambda t)\right)^{\frac{x}{\lambda}} \\
& =\frac{E i_{k}(\log (1+t))}{\log \left(1+\frac{1}{\lambda} \log (1+\lambda t)\right)}\left(1+\frac{1}{\lambda} \log (1+\lambda t)\right)^{\frac{x}{\lambda}} \\
& =\sum_{n=0}^{\infty} C_{n, \lambda, 2}^{(k)}(x) \frac{t^{n}}{n !} .
\end{aligned}
$$

Thus, by (3.6), we have the following theorem.

Theorem 3.1. Let $n \geq 0$ and $k \in \mathbb{Z}$, and $\Gamma(n)$ be a Gamma function. Then, we have

$$
C_{n, \lambda, 2}^{\left(k, \frac{1}{\Gamma}\right)}(x)=C_{n, \lambda, 2}^{(k)}(x)
$$

From (3.5), we get

$$
\begin{aligned}
& \sum_{n=0}^{\infty} C_{n, \lambda, 2}^{(k, p)} \frac{t^{n}}{n !}=\frac{u_{k}(\log (1+t) \mid p)}{\log \left(1+\frac{1}{\lambda} \log (1+\lambda t)\right)} \\
& =\frac{1}{\log \left(1+\frac{1}{\lambda} \log (1+\lambda t)\right)} \sum_{m=1}^{\infty} \frac{p(m)}{m^{k}(\log (1+t))^{m}} \\
& =\frac{1}{\log \left(1+\frac{1}{\lambda} \log (1+\lambda t)\right)} \sum_{m=0}^{\infty} \frac{p(m+1)(m+1) !}{(m+1)^{k}} \sum_{l=m+1}^{\infty} S_{1}(m+1, l) \frac{t^{l}}{l !} \\
& =\left(\sum_{j=0}^{\infty} C_{j, \lambda, 2} \frac{t^{j}}{j !}\right)\left(\sum_{l=0}^{\infty} \sum_{m=0}^{l} \frac{p(m+1)(m+1) !}{(m+1)^{k}} S_{1}(m+1, l) \frac{t^{l}}{l !}\right) \\
& =\sum_{n=0}^{\infty}\left(\sum_{l=0}^{\infty} \sum_{m=0}^{l}\left(\begin{array}{l}
n \\
l
\end{array}\right) \frac{p(m+1)(m+1) !}{(m+1)^{k}} \frac{S_{1}(m+1, l) C_{n-l, \lambda, 2}}{l+1}\right) \frac{t^{n}}{n !} .
\end{aligned}
$$

Therefore, by comparing the coefficients on both sides of (3.8), we obtain the following theorem. 
Theorem 3.2. Let $n \in \mathbb{N}$ and $k \in \mathbb{Z}$. Then we have

$$
C_{n, \lambda, 2}^{(k, p)}=\sum_{l=0}^{\infty} \sum_{m=0}^{l}\left(\begin{array}{l}
n \\
l
\end{array}\right) \frac{p(m+1)(m+1) !}{(m+1)^{k}} \frac{S_{1}(m+1, l) C_{n-l, \lambda, 2}}{l+1} .
$$

In particular,

$$
C_{n, \lambda, 2}^{\left(k, \frac{1}{\Gamma}\right)}=C_{n, \lambda, 2}^{(k)}=\sum_{l=0}^{\infty} \sum_{m=0}^{l}\left(\begin{array}{c}
n \\
l
\end{array}\right) \frac{S_{1}(m+1, l) C_{n-l, \lambda, 2}}{(m+1)^{k-1}(l+1)} .
$$

From (3.5), we observe that

$$
\begin{aligned}
& \sum_{n=0}^{\infty} C_{n, \lambda, 2}^{(k, p)}(x) \frac{t^{n}}{n !}=\frac{u_{k}(\log (1+t) \mid p)}{\log \left(1+\frac{1}{\lambda} \log (1+\lambda t)\right)}\left(1+\frac{1}{\lambda} \log (1+\lambda t)\right)^{\frac{x}{\lambda}} \\
& =\frac{u_{k}(\log (1+t) \mid p)}{\log \left(1+\frac{1}{\lambda} \log (1+\lambda t)\right)} \sum_{m=0}^{\infty}\left(\begin{array}{c}
\frac{x}{\lambda} \\
m
\end{array}\right)\left(\frac{1}{\lambda} \log (1+\lambda t)\right)^{m} \\
& =\left(\sum_{l=0}^{\infty} C_{l, \lambda, 2}^{(k, p)} \frac{t^{l}}{l !}\right)\left(\sum_{m=0}^{\infty}(x)_{m, \lambda} \lambda^{-2 m} \sum_{s=m}^{\infty} S_{1}(s, m) \frac{t^{s}}{s !}\right) \\
& =\left(\sum_{l=0}^{\infty} C_{l, \lambda, 2}^{(k, p)} \frac{t^{l}}{l !}\right)\left(\sum_{s=0}^{\infty} \sum_{m=0}^{s}(x)_{m, \lambda} \lambda^{-2 m} S_{1}(s, m) \frac{t^{s}}{s !}\right) \\
& =\sum_{n=0}^{\infty}\left(\sum_{l=0}^{n} \sum_{m=0}^{n-l} C_{l, \lambda, 2}^{(k, p)}(x)_{m, \lambda} \lambda^{-2 m} S_{1}(n-l, m)\right) \frac{t^{n}}{n !}
\end{aligned}
$$

From (3.11), we obtain the following theorem.

Theorem 3.3. Let $n \geq 0$ and $k \in \mathbb{Z}$. Then we have

$$
C_{n, \lambda, 2}^{(k, p)}(x)=\sum_{l=0}^{n} \sum_{m=0}^{n-l} C_{l, \lambda, 2}^{(k, p)}(x)_{m, \lambda} \lambda^{-2 m} S_{1}(n-l, m) .
$$


From (3.5), we observe that

$$
\begin{aligned}
& \sum_{n=0}^{\infty} C_{n, \lambda, 2}^{(k, p)} \frac{t^{n}}{n !} \\
& =\frac{u_{k}(\log (1+t) \mid p)}{\log \left(1+\frac{1}{\lambda} \log (1+\lambda t)\right)} \\
& =\frac{1}{\log \left(1+\frac{1}{\lambda} \log (1+\lambda t)\right)} \sum_{m=0}^{\infty} \frac{p(m+1)}{(m+1)^{k}} \frac{m !}{m !}(\log (1+t))^{m+1} \\
& =\frac{\log (1+t)}{\log \left(1+\frac{1}{\lambda} \log (1+\lambda t)\right)} \sum_{m=0}^{\infty} \frac{p(m+1)}{(m+1)^{k}} \frac{m !}{m !}(\log (1+t))^{m} \\
& =\frac{\log (1+t)}{\log \left(1+\frac{1}{\lambda} \log (1+\lambda t)\right)} \sum_{m=0}^{\infty} \frac{p(m+1)}{(m+1)^{k}} \frac{m !}{m !}(\log (1+t))^{m} \\
& =\frac{\log (1+t)}{t} \frac{t}{\log \left(1+\frac{1}{\lambda} \log (1+\lambda t)\right)} \sum_{m=0}^{\infty} \frac{p(m+1) m !}{(m+1)^{k}} \sum_{l=m}^{\infty} S_{1}(l, m) \frac{t^{l}}{l !} \\
& =\left(\sum_{s=0}^{\infty} D_{s} \frac{t^{s}}{s !}\right)\left(\sum_{a=0}^{\infty} C_{a, \lambda, 2} \frac{t^{a}}{a !}\right)\left(\sum_{l=0}^{\infty} \sum_{m=0}^{l} \frac{p(m+1) m !}{(m+1)^{k}} S_{1}(l, m) \frac{t^{l}}{l !}\right) \\
& =\left(\sum_{b=0}^{\infty} \sum_{a=0}^{b}\left(\begin{array}{l}
b \\
a
\end{array}\right) D_{b-a} C_{a, \lambda, 2} \frac{t^{b}}{b !}\right)\left(\sum_{l=0}^{\infty} \sum_{m=0}^{l} \frac{p(m+1) m !}{(m+1)^{k}} S_{1}(l, m) \frac{t^{l}}{l !}\right) \\
& =\sum_{n=0}^{\infty}\left(\sum_{l=0}^{n} \sum_{a=0}^{n-l} \sum_{m=0}^{l}\left(\begin{array}{l}
n \\
l
\end{array}\right) D_{n-l-a} C_{a, \lambda, 2} \frac{p(m+1) m !}{(m+1)^{k}} S_{1}(l, m)\right) \frac{t^{n}}{n !}
\end{aligned}
$$

By comparing coefficients on both sides of (3.13), we obtain the following theorem.

Theorem 3.4. Let $n \geq 0$ and $k \in \mathbb{Z}$. Then we have

$$
C_{n, \lambda, 2}^{(k, p)}=\sum_{l=0}^{n} \sum_{a=0}^{n-l} \sum_{m=0}^{l}\left(\begin{array}{l}
n \\
l
\end{array}\right) D_{n-l-a} C_{a, \lambda, 2} \frac{p(m+1) m !}{(m+1)^{k}} S_{1}(l . m) .
$$

\section{Conclusions}

In 2019 Kim-Kim considered the polyexponential functions and poly-Bernoulli polynomials and Kim [14] introduced the degenerate Cauchy numbers of the second kind. In the same view as these functions and polynomials, we defined the degenerate poly-Cauchy polynomials of the second kind (Eq.(2.3)) and obtained some identities of the degenerate poly-Cauchy numbers of the second kind (Theorems 2.1, and 2.2). In particular, we obtained an identity of the degenerate poly-Cauchy polynomials of the second kind in Theorem 2.3. Furthermore, by using the unipoly functions, we defined the degenerate unipoly-Cauchy polynomials of the second kind(Eq. (3.5)) and obtained some properties of the degenerate unipoly-Cauchy numbers of the second kind(Theorems 3.1, and 3.3). Finally, we obtained an identity of the degenerate unipoly-Cauchy polynomials of the second kind in Theorem 3.3 and gave the identity indicating the relationship of the degenerate unipoly-Cauchy numbers of the second 
kind and the Daehee numbers and degenerate Cauchy numbers of the second kind in Theorem 3.4.

LCJ conceived of the framework and structured the whole paper; HKK and LCJ checked the results of the paper and completed the revision of the article. All authors have read and agreed to the published version of the manuscript.

Supported by the Basic Science Research Program, the National Research Foundation of Korea, the Ministry of Education, (NRF-2018R1D1A1B07049584)..

The authors declare that they have no competing interests.

\section{References}

1. B. S. Borisov, The $p$-binomial transform Cauchy numbers and figurate numbers, Proc. Jangjeon Math. Soc. 19(4),(2016), 631-644.

2. S.-K. Chung, G.-W. Jang, D. S. Kim and J. Kwon, Some identities of the type 2 degenerate Bernoulli and Euler numbers, Adv. Stud. Contemp. Math. (Kyungshang) 29(4),(2019), 613632 .

3. U. Duran, M. Acikgoz, S. Araci, Hermite based poly-Bernoulli polynomials with a q-parameter, Adv. Stud. Contemp. Math. (Kyungshang) 28(2),(2018), 285-296.

4. L.C. Jang, W. Kim, H.-I. Kwon, T. Kim, On degenerate Daehee polynomials and numbers of the third kind, J. Comput. Appl. Math. 364, (2020), 112343, 9 pp.

5. D.S. Kim, T. Kim, A note on polyexponential and unipoly functions, Russ. J. Math. Phys. 26(1),(2019), 40-49.

6. D. S. Kim, T. Kim, On sums of finite products of balancing polynomials, Comput. Appl. Math. 377, (2020), 112913.

7. T. Kim, D. S. Kim, H. Y. Kim, L.C.Jang, Degenerate poly-Bernoulli numbers and polynomials, Informatica, 31(3),(2020), 2-8.

8. T. Kim, D. S. Kim, D. V. Dolgy, J. Kwon, Some identities on generalized degenerate Genocchi and Euler numbers, Informatica, 31(4),(2020), 42-51.

9. T. Kim, D. S. Kim, J. Kwon, H. Lee, A note on degenerate gamma random variables, Revista de Educacion 388(4),(2020), 39-44.

10. T. Kim, D.S. Kim, Some identities of extended degenerate r-central Bell polynomials arising from umbral calculus, Rev. R. Acad. Cienc. Exactas Fs. Nat. Ser. A Mat. RACSAM 114(1),(2020), 19pp.

11. T. Kim, D.S. Kim, H. Lee, J. Kwon, Degenerate binomial coefficients and degenerate hypergeometric functions, Adv. Difference Equ. 2020:115,(2020), 17pp.

12. T. Kim, D. S. Kim, Degenerate polyexponential functions and degenerate Bell polynomials, J. Math. Anal. Appl. 487(2),(2020),124017.

13. T. Kim, D. S. Kim, L.C. Jang, H. Y. Kim, A note on discrete degenerate random variables,, Proc. Jangjeon Math. Soc. 23(1),(2020), 125-135.

14. T. Kim, Degenerate Cauchy numbers and polynomials of the second kind, Adv. Stud. Contemp. Math. (Kyungshang) 27,(2017), 441-449.

15. T. Kim, On degenerate Cauchy numbers and polynomials, Proc. Jangjeon Math. Soc. 18(3),(2015), 307-312.

16. M. Kaneko, Poly-Bernoulli numbers, J. Theor. Nombres Bordeanes 9(1),(1997), 221-228.

17. W. A. Khan and M. Ahmad, Partially degenerate poly-Bernoulli polynomials associated with Hermite polynomials, Adv. Stud. Contemp. Math. (Kyungshang) 28(3),(2018), 487-496.

18. W. A. Khan, A new class of degenerate Frobenius-Euler-Hermite polynomials, Adv. Stud. Contemp. Math. (Kyungshang) 28(4),(2018), 567-576.

19. T. Komatsu, Higher-order convolution identities for Cauchy numbers of the second kind,Proc. Jangjeon Math. Soc. 18(3),(2015), 369-383.

20. D. V. Kruchinin, V. V. Kruchinin, Explicit formula for reciprocal generating function and its application, Adv. Stud. Contemp. Math. (Kyungshang) 29(3),(2019), 365-372 
21. W. A. Khan, Some identities for degenerate complete and incomplete $r$-Bell polynomials, J. Inequal. Appl. 2020:23 ,(2020), 9pp.

22. D. Lim, Modified degenerate Daehee numbers and polynomials arising from differential equations, Adv. Stud. Contemp. Math. (Kyungshang) 28(3) ,(2018), 497-506

23. S.-S. Pyo, Degenerate Cauchy numbers and polynomials of the fourth kind, Adv. Stud. Contemp. Math. (Kyungshang) 28(1),(2018), 127-138

24. S. -S. Pyo, T. Kim, S. -H. Rim, Degenerate Cauchy numbers of the third kind, J. Ineq. Appl. 2018:32,(2018), 12pp.

25. S. Roman, The umbral calculus, Pure and Applied Mathematics, 111. Academic Press, Inc. [Harcourt Brace Jovanovich, Publishers], New York, 1984. x+193 pp. ISBN: 0-12-594380-6

1 Department of Mathematics Education, Daegu Catholic University, Gyeongsan 38430, RePublic of KoreA

E-mail address: hkkim@cu.ac.kr

2 Graduate School of Educhtion, Konkuk University, Seoul, 05029, Republic of KOREA, *CORRESPONDING AUTHOR

E-mail address: Lcjang@konkuk.ac.kr 